\title{
Pregnancy outcomes of infertile women with co- existing endometriosis and adenomyosis after laparoscopic surgery: a long-term follow-up
}

Jinghua Shi

Peking Union Medical College Hospital https://orcid.org/0000-0001-5267-9092

Yi Dai

Peking Union Medical College Hospital

Junji Zhang

Peking Union Medical College Hospital

Xiaoyan Li

Peking Union Medical College Hospital

Shuangzhen Jia

Peking Union Medical College Hospital

Jinhua Leng ( $\boldsymbol{\nabla}$ lengjenny@vip.sina.com )

Peking Union Medical College Hospital

Research article

Keywords: Adenomyosis, Endometriosis; Infertility, Laparoscopic surgery, Pregnancy outcome

Posted Date: October 6th, 2020

DOl: https://doi.org/10.21203/rs.3.rs-85435/v1

License: (9) (i) This work is licensed under a Creative Commons Attribution 4.0 International License.

Read Full License 


\section{Abstract}

Background: Adenomyosis and endometriosis are often co-existent. Laparoscopic surgery is one of the main methods to diagnose and treat these conditions. However, very few studies have been done that concentrate on the pregnancy outcomes of infertile women with both adenomyosis and endometriosis after laparoscopic surgery, as well as the relevant influential factors.

Methods: This is a retrospective, cross-sectional study including infertile women diagnosed with endometriosis and adenomyosis. All patients had undergone laparoscopic surgery and were divided into two groups according to pregnancy outcomes. Demographic data, operation records, and pregnancy outcomes were collected.

Results: Ninety-seven patients had live births, including 81 full-term and 16 preterm deliveries. The biochemical pregnancy, clinical pregnancy, and live birth rates were $80.87 \%, 67.4$, and $55.11 \%$ respectively. One hundred thirty-five patients received IVF with 70 (51.85\%) patients having live births. Age, size of endometrioma, and size of uterus were significantly lower in those who had a successful delivery. There was no statistical difference in symptoms except anemia $(13.40 \%$ vs. $25.32 \%, p=0.044)$. The group that failed to have a live birth had a higher percentage of ovarian and peritoneal endometriosis $(P<.05)$, while the distribution of deep infiltrating endometriosis and adenomyosis types were similar. Mean uterus diameter (OR: $0.636,95 \% \mathrm{Cl}: 0.434-0.932, \mathrm{p}=0.020$ ) and endometriosis fertility index (EFI) (OR:1.299, 95\% Cl: $1.101-1.531, p=0.002$ ) were significantly correlated with live births in the multivariate analysis.

Conclusions: Endometriosis and adenomyosis have an adverse effect on fertility. IVF is an important technology improving pregnancy rate even after surgery. The size of the uterus and EFI were independent risk factors for pregnancy outcomes.

\section{Introduction}

Adenomyosis (AM) and endometriosis (EM) are benign conditions of the uterus, defined by the presence of endometrial glands and stroma within the myometrium and outside of the uterus. Histological diagnosis is the most accurate method to identify these conditions, but they can also be diagnosed clinically when patients are presenting with symptoms. These symptoms include dysmenorrhea, dyspareunia, abnormal uterine bleeding, and infertility. AM shares some pathogenic mechanisms with EM[1]. Our center reported that $39.9 \%$ of women with endometrioma also had AM[2], while $33.3 \%[3]$ of 72 patients histologically diagnosed with AM had concomitant EM. Chapron et al. [4] reported that the coexistence rate could be as high as $87.4 \%$.

AM and EM both have adverse effects on fertility, making treatment more complicated. In addition to the impacts on reproductive performance (infertility and miscarriage) and pregnancy outcomes (preterm labor, fetal growth restriction, placenta previa and even uterine rupture) are also affected by $\operatorname{AM}[5,6]$ and $\mathrm{EM}[7]$. 
Laparoscopic surgery is one of the main ways to diagnose as well as treat these uterine conditions, but it is difficult to predict pregnancy outcomes in infertile women diagnosed with both AM and EM. The endometriosis fertility index $(\mathrm{EFI})$ has been proven to be a useful model to predict pregnancy outcomes[8]. However, adenomyosis is not included. Additionally, the effect of the removal of coexisting adenomyosis on fertility outcomes is not fully evaluated and few studies have specifically concentrated on this field. In this study we aimed to explore pregnancy outcomes and use long term follow up to analyze factors relating to fertility outcomes of infertile women with both adenomyosis and endometriosis after laparoscopic surgery.

\section{Material And Methods}

\section{Ethical approval and informed consent for study}

This study was approved by the Ethics Committee of Peking Union Medical College Hospital (S-K1055), and all procedures involving human participants followed the ethical standards of the IRB from the study center. Informed consent was obtained from all patients at their follow-up interviews.

\section{Patient population}

We identified all the patients who underwent laparoscopic surgery for the first time in our hospital diagnosed with both EM and AM between January 2013 and December 2017. Among them, 226 patients were infertile and wished to conceive after surgery. During the surgery, endometriomas were removed and peritoneal endometrial tissue was coagulated. Deep infiltrating endometriosis and adenomyoma were resected if patients had severe symptoms or repeated IVF-ET failure due to unknown reasons. Presurgical symptoms and surgical data, including postoperative complications, were retrieved from patient admission and operative databases. The revised American Fertility Society (rAFS) score revised American Society for Reproductive Medicine (rASRM) staging, and EFI score were collected according to surgical records and infertility history. Post-surgical symptoms and pregnancy outcomes were collected from follow up interviews with outpatients.

Ultrasonographic exams were performed after the menstrual cycle that occurred most recently before surgery. Features on ultrasounds that suggested AM are as follows: asymmetrical myometrial thickening, myometrial cysts, linear striations, hyperechoic islands, or an irregular and thickened endometrialmyometrial junctional zone[9]. If more than two features were present and the lesions were located in only one part of the uterine wall, a diagnosis of focal AM was made. Diffuse AM was diagnosed as lesions in more than one site within the myometrium, more often being widely spread rather than forming a confined lesion[10]. Endometriosis was visually inspected by laparoscopy and histologically confirmed. Deep infiltrating endometriosis was diagnosed as the presence of one or more endometriotic nodules deeper than $5 \mathrm{~mm}$.

The exclusion criteria were as follows: (1) age $<20$ or $>40$ years; (2) patient underwent a bilateral oophorectomy or hysterectomy; (3) intraoperative conversion to a laparotomy; (4) concomitant diseases 
that clearly affect fertility, such as submucosal fibroids, premature ovarian failure, systemic lupus erythematosus, malignant tumors, etc.

\section{Statistical analysis}

Continuous data were presented as median (interquartile range) or mean $\pm S D$ and compared using t-tests or ANOVA tests. Categorical data were described by the number of cases (including percentages) and compared with Fisher's exact or chi-square tests. Potential risk factors $(p<0.2)$ were identified using univariate analysis and multivariate, logistic regression analysis (Stepwise). The odds ratio (OR) and 95\% confidence interval $(\mathrm{Cl})$ were calculated as a measure of the impact on live births. All analyses used a two-tailed a of 0.05 and were performed using software SPSS (Version 20.0, IBM Corp., Armonk, NY, USA).

\section{Results}

\section{Patient characteristics}

During the study period, 226 infertile patients with AM and EM were identified. 43 patients were excluded due to an elderly age $(n=32)$ or insufficient follow-up data $(n=11)$. Here we define a successful delivery as the patient having a live birth. To report the factors associated with successful live births, we divided the 176 women into two groups: Group $A(n=97)$ contained 81 full-term infants and 16 preterm infants, and Group B $(\mathrm{n}=79)$ contained those who didn't get live birth. The remaining seven patients were still pregnant and were excluded. Details regarding the flowchart of the group selection process for the patients are provided in Fig. 1. Basic characteristics of both groups are presented in Table 1. 
Table 1

Population characteristics of the live birth and non-live birth group

\begin{tabular}{|c|c|c|c|}
\hline & Group A & Group B & p \\
\hline & $\begin{array}{l}\text { Women who had a } \\
\text { successful delivery }(n=97)\end{array}$ & $\begin{array}{l}\text { Women who did not have a } \\
\text { successful delivery }(n=79)\end{array}$ & \\
\hline Age (years) & $32.52 \pm 3.20$ & $33.53 \pm 3.95$ & $0.046 *$ \\
\hline $\mathrm{BMI}\left(\mathrm{kg} / \mathrm{m}^{2}\right)$ & $21.74 \pm 2.78$ & $20.51 \pm 4.72$ & $0.033^{*}$ \\
\hline Type of Infertility & 60 & 52 & NS \\
\hline Primary infertility & 37 & 27 & \\
\hline Secondary infertility & & & \\
\hline Gravida & $0(0-5)$ & $0(0-5)$ & NS \\
\hline Parity & $0(0-1)$ & $0(0-2)$ & NS \\
\hline Abortion & $19(19.58 \%)$ & $23(29.11 \%)$ & NS \\
\hline Previous ART & $21(21.65 \%)$ & $14(17.72 \%)$ & NS \\
\hline $\begin{array}{l}\text { Duration of } \\
\text { infertility(years) }\end{array}$ & $3(1-8)$ & $2(1-11)$ & NS \\
\hline Length of period(day) & $5.88 \pm 1.86$ & $6.22 \pm 2.04$ & NS \\
\hline Menstrual cycle(day) & $28.94 \pm 6.17$ & $28.94 \pm 9.35$ & NS \\
\hline $\begin{array}{l}\text { Duration of } \\
\text { dysmenorrheal(years) }\end{array}$ & $5.33 \pm 5.77$ & $6.30 \pm 6.29$ & NS \\
\hline Dysmenorrhea (VAS) & $4.22 \pm 3.59$ & $4.58 \pm 3.49$ & NS \\
\hline Anemia & $13(13.40 \%)$ & $20(25.32 \%)$ & $0.044^{*}$ \\
\hline CA125(U/ml) & $80.67 \pm 92.47$ & $109.57 \pm 98.48$ & NS \\
\hline $\mathrm{FSH}(\mathrm{IU} / \mathrm{I})$ & $7.12 \pm 2.21$ & $8.43 \pm 2.81$ & $0.005^{\star}$ \\
\hline $\mathrm{E} 2(\mathrm{pg} / \mathrm{ml})$ & $53.69 \pm 41.14$ & $48.88 \pm 21.85$ & NS \\
\hline $\begin{array}{l}\text { Mean uterus diameter } \\
(\mathrm{cm})\end{array}$ & $4.91 \pm 0.79$ & $5.42 \pm 1.20$ & $0.001 *$ \\
\hline $\begin{array}{l}\text { Mean cyst diameter } \\
\text { (cm) }\end{array}$ & $2.63 \pm 3.43$ & $4.38 \pm 4.00$ & $0.002 *$ \\
\hline $\begin{array}{l}\text { Note:Data are presente } \\
\text { assisted reproductive } \mathrm{t} \\
\text { stimulating hormone; } \mathrm{E}\end{array}$ & $\begin{array}{l}\text { s mean } \pm \text { SD or } n(\%) ; N S, n \\
\text { nology; VAS, visual analog } \\
\text { estrogen. }\end{array}$ & $\begin{array}{l}\text { gnificant; BMI, body mass i } \\
\text {; CA125,cancer antigen } 12\end{array}$ & icle \\
\hline
\end{tabular}




\section{Surgical findings}

Compared to the women who did not have a successful delivery, the women who had a successful delivery had significantly lower rAFS scores $(33.62 \pm 33.53$ vs. $47.56 \pm 38.22, p=0.011)$ and higher EFI scores $(6.87 \pm 2.04$ vs. $5.67 \pm 2.09, p=0.001)$. Blood loss during the laparoscopy in group A was also less than group $B(46.03 \pm 51.80 \mathrm{ml}$ vs $84.08 \pm 114.39 \mathrm{ml}, \mathrm{p}=0.009)$ and so was surgical time $(56.60 \pm$ $21.42 \mathrm{~min}$ vs. $65.76 \pm 27.34 \mathrm{~min}, p=0.022$ ). This study also found that the non-live birth group had a significantly higher proportion of OEM (ovarian endometriosis) and PEM (peritoneal endometriosis). However, there were no statistical difference in DIE (deep infiltrating endometriosis), leiomyoma, endometrial polyps, or obstruction of oviduct (Table 2).

Table 2

Surgical findings between live birth and non-live birth groups

\begin{tabular}{|llll|}
\hline & Group A(n= 97) & Group B(n= 79) & p value \\
\hline Ovarian endometriosis & $49(50.52 \%)$ & $57(72.15 \%)$ & $0.004^{*}$ \\
\hline Peritoneal endometriosis & $65(67.01 \%)$ & $40(72.15 \%)$ & $0.028^{*}$ \\
\hline $\begin{array}{l}\text { Deep infiltrating } \\
\text { endometriosis }\end{array}$ & $35(36.08 \%)$ & $22(27.85 \%)$ & NS \\
AM type & $52(53.61 \%)$ & $43(54.43 \%)$ & NS \\
Diffuse & $45(46.39 \%)$ & $36(45.57 \%)$ & \\
Local & & & \\
\hline Leiomyoma & $29(29.90 \%)$ & $18(22.78 \%)$ & NS \\
\hline Obstruction of oviduct & $22(22.68 \%)$ & $23(29.11 \%)$ & NS \\
\hline Endometrial polyps & $31(31.96 \%)$ & $25(31.64 \%)$ & NS \\
\hline Note:Data are presented as mean \pm SD or $n(\%) ;$ NS, not significant. \\
\hline
\end{tabular}

\section{Fertility results and pregnancy outcomes}

After a long term follow up (median 47 months, range $20-80$ months), 35 (19.13\%) patients failed to conceive and $13(7.10 \%)$ patients stopped trying to conceive: four due to disease relapses, two due to cases of depression, four due to premature ovarian failures (POF), one case due to endometrial intraepithelial neoplasia (EIN), and two due to repeated in vitro fertilization (IVF) failures. One hundred thirty five $(73.77 \%)$ patients received IVF, among which four patients lacked follow up data and 70 patients had live births $(51.85 \%)$. The mean time from surgery to pregnancy was $12.89 \pm 8.66$ months and the mean time from stopping contraception after surgery to pregnancy was $8.56 \pm 8.48$ months.148(80.87\%) patients got a positive pregnancy test, clinical pregnancy rate, or presence of a fetal 
heartbeat at 12 weeks of gestation, was $67.4 \%$, and $55.11 \%$ ultimately achieved a successful delivery. Forty $(27.03 \%)$ patients experienced natural abortions, two (1.35\%) had an ectopic pregnancy, and five (3.38\%) had intrauterine deaths (fetal death at or after the 20th week of gestation). Ninety five (51.91\%) patients took their babies home, among which 16 (16.84\%) had preterm labor and $12(12.63 \%)$ had abnormal placentae (seven placenta previa, four placenta acrreta, and one placenta increta).

To determine the factors associated with a successful delivery, univariate analysis and multivariate logistic regression analysis were performed. Variables showing a tendency of association with live birth rates $(p$ values $<0.20)$ in the univariate analysis were included in the multivariate model. When two variables were highly correlated, we introduced only one of them into the model and suppressed the other; for example when examining age and FSH levels, the latter was suppressed. Surgical time $(p=0.016)$ and blood loss $(p=0.011)$ showed a tendency of association, but they were excluded as they were highly correlated with the AFS score and type of AM. EFI includes age, infertility type, years of infertility, function of tube/fimbria/ovaries, and AFS and therefore, they wouldn't be included as candidate factors. EFI ( $p=$ $0.00)$, anemia $(p=0.047)$,VAS $(p=0.112)$, CA125 $(p=0.093)$, mean uterine diameter $(p=0.002)$, assisted reproductive technology (ART) $(p=0.122)$, and medical history $(p=0.017)$ were included as covariates in the final model. AM type $(p=0.159)$ and AM surgery $(p=0.110)$ were also included as they were reported to be related to the pregnancy rate[11]. Table 3 presents the estimated ORs with the standard errors (SE) and $95 \%$ Cls between live birth rates and significantly relevant confounders. Other variables had no significant relationship with live births.

Table 3

Significant prognostic factors of live birth rate after multivariate analysis

\begin{tabular}{|lllll|}
\hline Characteristics & OR & SE & $\mathbf{9 5 \%}$ Cl & P-value \\
\hline Mean uterus diameter & 0.636 & 0.195 & $0.434-0.932$ & $0.020^{*}$ \\
\hline EFI & 1.299 & 0.084 & $1.101-1.531$ & $0.002 *$ \\
\hline Note: OR: odds ratio; SE: standard error; Cl: confidential interval \\
\hline
\end{tabular}

\section{Discussion}

In this study, 176 infertile women with both EM and AM had laparoscopic surgery and completed an outpatient follow up.

Few studies have concentrated on the pregnancy outcomes of women with both AM and EM; most research reported only EM or AM patients or grouped the two diseases together. Chloé Maignien et al.[12] studied 359 infertile EM patients and the pregnancy and live birth rates were $44 \%$ and $32 \%$, respectively. Our results were a bit higher, perhaps because of the longevity of our follow up. Some patients were able to have a natural live birth even after repeated IVF-ET failure. 
EM phenotypes seemed to impact pregnancy outcomes, as there were significant differences between the live-birth group and stillbirth group. Our previous results verified the presence of follicles in the cyst walls of the endometrioma in paraffin sections[13] and that FSH levels were significantly decreased after laparoscopic bilateral ovary endometrioma resection[14]. Vercellini et al. retrospectively analyzed 419 patients who achieved natural conception and divided them into four groups according to the type of EM. The total pregnancy loss rate was $20.8 \%$, which was close to ours $(20.03 \%)$. They also observed a higher miscarriage rate in women with ovarian endometriomas (26\%) than in those with the peritoneal type (12\%)[15]. However, these findings were contrary to the report done by Chloé Maignien et al.[12]. In his study, the live birth rates per cycle were $13.7 \%, 16.5 \%$ and $16 \%$ in peritoneal, ovarian, and deep infiltrating EM subgroups $(p=0.82)$, respectively.

In addition to the reduced oocyte yield, reduced fertilization rate (FR), and increased miscarriage rate, AM and EM were also reported to be related to several obstetrical and fetal complications. However, the evidence is still scanty and conflicting. A meta-analysis in 2019[16] including 104 papers demonstrated that EM could be associated with preterm delivery (OR 1.38, $\mathrm{Cl} 1.01-1.89$ ), caesarean section delivery (OR $1.98 \mathrm{Cl} 1.64-2.38$ ), and neonatal unit admission following delivery (OR 1.29, $\mathrm{Cl} 1.07-1.55$ ). It is noteworthy that no OR exceeded three. Two other systematic reviews failed to draw conclusions on obstetrical complications related to EM except for preterm delivery[17] and placenta previa[18]. The results of this study showed a preterm birth rate of $16.84 \%$ and placenta previa rate of $7.36 \%$, which are findings slightly higher than in Benaglia L's report. In his retrospective, matched, case-control study[19], the rate of preterm birth was similar between the EM and non-EM groups ( $14 \%$ and $14 \%$, respectively, $p=$ 0.89), while placenta previa was more common in women with EM than in the control group (6\% versus $1 \%$, respectively; $p=0.006$, OR $4.8,95 \% \mathrm{Cl}$ : $1.4-17.2$ ). A possible explanation for this might be that our cases had patients with both EM and AM. In a multicenter, retrospective, questionnaire survey[20] including 272 pregnant women with AM from 65 facilities, the preterm delivery rate was reported to be as high as $24.4 \%$. Several studies further analyzed the clinical factors that affect pregnancy outcomes. In a retrospective study of 631 women with EM who became pregnant by ART [21], the reported incidence of preterm delivery or abnormal placental positioning didn't increase in stages I-III, but increased significantly in stage IV. Dr. Kim and colleagues[22] reported a preterm labor rate of $24.56 \%$ in a retrospective study and found that uterine wall thickness in the second trimester was related to subsequent preterm delivery. In our study, the size of uterus was also found to be significantly different between the two groups.

The etiology of infertility might include disordered inflammatory factors like prostaglandin (PG) production (PGE2 and PGF2a), cyclooxygenase 2 (COX-2)[23], abnormal ER- and PR-mediated signaling pathways associated with progesterone resistance[24], impaired trophoblast invasiveness, and uterine contractility[25]. These can also be related to failure of the physiologic transformation of spiral arteries in the inner myometrial segment, or junctional zone (JZ). Alterations of the JZ in women with EM and AM can influence vascular resistance of JZ spiral arteries at the onset of decidualization[25] and lead to incomplete spiral artery remodeling and the reduction of placental blood flow[26]. The mechanism is 
complex, and the existing results are debatable. The influence of co-existing EM and AM on pregnancy is not simply superimposed, but rather the two diseases interact with each other.

The strength of this study is its diagnosis. The golden standard of AM diagnosis is based on histological specimens, which is an invasive technique and has a potential of uterine rupture during pregnancy and delivery. Therefore, non-invasive technics like ultrasound and MRI are preferred. MRI, with a sensitivity from $78-88 \%$ and specificity from $67-93 \%$ [27], was traditionally considered to be more accurate than ultrasound. However, with the development of high-quality transvaginal ultrasound (TVUS), a systematic review including 10 studies (1168 records) proved that the two techniques are comparable[28]. The accuracy of the ultrasound in AM diagnoses is high with a mean sensitivity of 0.72 (95\% Cl: $0.65-0.79$ ), specificity of 0.81 (95\% Cl: $0.77-0.85$ ), and area under the curve (AUC) of $0.85[29,30]$. Additionally, ultrasounds are much cheaper and more convenient. The diagnoses of EM and its subtypes are based on laparoscopic surgery, which is the gold standard and quite reliable. However, women referred to our hospital may have had particularly severe forms of AM, EM, or repeated IVF failure, which introduces potential selection and comparison bias. Second, the follow up didn't include an analysis of IVF details. Most patients went to their local ART center for controlled ovarian stimulation and IVF-ET and could hardly recall the details, remembering only the final results. There are several other limitations to these results. First, our study is retrospective. Second, the number of cases was limited and we could not perform subtype analyses according to the obstetric complications. Third, other disputed factors were not included, such as the excision of DIE[31], use of GnRHa[32], and complete removal of endometrial lesions [33]. Fourth, this study only includes patients that needed laparoscopic surgery. Although dysmenorrhea, metrorrhagia, chronic pelvic pain, dyspareunia, and infertility often occur, a third of the women were asymptomatic[27] and we are worried about the extent to which this will affect our fertility and sterility rates.

\section{Conclusions}

Overall, our study suggests that co-existing EM and AM has adverse effects on both reproductive performance and the outcome of pregnancy. Age, BMI, and size of endometrioma and the uterus were related to the prognosis. IVF is an important technique in improving the pregnancy rate following surgery. These findings may prove useful in daily practice for offering optimal infertility consultation to women with both EM and AM. Further prospective studies with larger sample sizes are still needed to draw firmer conclusions.

\section{Declarations}

\section{Acknowledgments}

We appreciate the whole stuff at Peking Union Medical College Hospital (PUMCH) for their clinical work and data recording about the cases we reported in this article. 


\section{Author contribution}

JH-S and JH-L designed the study. XY-L, SZ-J, and J-J Zhang retrieved the data and performed the data analysis. JH-S and Y-D drafted the manuscript. All the authors participated in the discussion of the results and critically revised the manuscript.

\section{Compliance with Ethical Standards:}

\section{Funding:}

This study was funded by the National Key R\&D Program of China (No. 2017YFC1001200), National Natural Science Foundation of China (No. 81541016)

\section{Conflict of interest:}

All authors declared that they have no conflict of interest related to the topic to declare.

\section{Ethical approval:}

All procedures performed in studies involving human participants were in accordance with the ethical standards of the institutional and/or national research committee and with the 1964 Helsinki declaration and its later amendments or comparable ethical standards.

\section{Informed consent:}

Informed consent was obtained from all individual participants included in the study.

\section{References}

1. Brosens, I., G. Kunz, and G. Benagiano, Is adenomyosis the neglected phenotype of an endomyometrial dysfunction syndrome? Gynecol Surg, 2012. 9(2): p. 131-137.

2. Li, X.Y., et al., Risk factors for postoperative recurrence of ovarian endometriosis: long-term follow-up of 358 women. J Ovarian Res, 2019. 12(1): p. 79.

3. Lv, Y., et al., The medium to long term clinical outcomes of laparoscopic conservative surgery on adenomyomas. CHINESE JOURNAL OF PRACTICAL GYNECOLOGY AND OBSTETRICS, 2011. 27(10): p. $753-756$.

4. Chapron, C., et al., Relationship between the magnetic resonance imaging appearance of adenomyosis and endometriosis phenotypes. Hum Reprod, 2017. 32(7): p. 1393-1401.

5. Mochimaru, A., et al., Adverse pregnancy outcomes associated with adenomyosis with uterine enlargement. Journal of Obstetrics and Gynaecology Research, 2014. 41.

6. Razavi, M., et al., Systematic review and meta-analysis of adverse pregnancy outcomes after uterine adenomyosis. Int J Gynaecol Obstet, 2019. 145(2): p. 149-157. 
7. Harada, T., et al., Adverse obstetrical outcomes for women with endometriosis and adenomyosis: A large cohort of the Japan Environment and Children's Study. PLoS One, 2019. 14(8): p. e0220256.

8. Maheux-Lacroix, S., et al., Endometriosis fertility index predicts live births following surgical resection of moderate and severe endometriosis. Hum Reprod, 2017. 32(11): p. 2243-2249.

9. Lazzeri, L., et al., Preoperative and Postoperative Clinical and Transvaginal Ultrasound Findings of Adenomyosis in Patients With Deep Infiltrating Endometriosis. Reprod Sci, 2014. 21(8): p. 1027-1033.

10. Van den Bosch, T., et al., Terms, definitions and measurements to describe sonographic features of myometrium and uterine masses: a consensus opinion from the Morphological Uterus Sonographic Assessment (MUSA) group. Ultrasound Obstet Gynecol, 2015. 46(3): p. 284-98.

11. Tan, J., et al., Reproductive Outcomes after Fertility-Sparing Surgery for Focal and Diffuse Adenomyosis: A Systematic Review. J Minim Invasive Gynecol, 2018. 25(4): p. 608-621.

12. Maignien, C., et al., Prognostic factors for assisted reproductive technology in women with endometriosis-related infertility. Am J Obstet Gynecol, 2017. 216(3): p. 280.e1-280.e9.

13. Shi, J., et al., Follicle loss after laparoscopic treatment of ovarian endometriotic cysts. Int J Gynaecol Obstet, 2011. 115(3): p. 277-81.

14. Shi, J.H., et al., Influence of Laparoscopic Cystectomy on Ovarian Reserve Function and Pregnantic Outcome in Women with Ovarian Endometriotic Cyst. MEDICAL JOURNAL OF PEKING UNION MEDICAL COLLEGE HOSPITAL, 2011. 002(2): p. 20-24.

15. Vercellini, P., et al., Pregnancy outcome in women with peritoneal, ovarian and rectovaginal endometriosis: a retrospective cohort study. Bjog, 2012. 119(12): p. 1538-43.

16. Horton, J., et al., Reproductive, obstetric, and perinatal outcomes of women with adenomyosis and endometriosis: a systematic review and meta-analysis. Human reproduction update, 2019. 25.

17. Vigano, P., L. Corti, and N. Berlanda, Beyond infertility: obstetrical and postpartum complications associated with endometriosis and adenomyosis. Fertil Steril, 2015. 104(4): p. 802-812.

18. Leone Roberti Maggiore, U., et al., A systematic review on endometriosis during pregnancy: diagnosis, misdiagnosis, complications and outcomes. Human Reproduction Update, 2015. 22(1): p. 70-103.

19. Benaglia, L., et al., Pregnancy outcome in women with endometriosis achieving pregnancy with IVF. Hum Reprod, 2016. 31(12): p. 2730-2736.

20. Tamura, H., et al., Complications and outcomes of pregnant women with adenomyosis in Japan. Reprod Med Biol, 2017. 16(4): p. 330-336.

21. Fujii, T., et al., Assisted reproductive technology pregnancy complications are significantly associated with endometriosis severity before conception: a retrospective cohort study. 14(1): p. 73.

22. Kim, Y.-M., et al., Uterine wall thickness at the second trimester can predict subsequent preterm delivery in pregnancies with adenomyosis. Taiwanese Journal of Obstetrics and Gynecology, 2019. 58: p. 598-603.

23. Vannuccini, S., et al., Infertility and reproductive disorders: impact of hormonal and inflammatory mechanisms on pregnancy outcome. Hum Reprod Update, 2016. 22(1): p. 104-15. 
24. Lessey, B.A. and S.L. Young, Homeostasis imbalance in the endometrium of women with implantation defects: the role of estrogen and progesterone. Semin Reprod Med, 2014. 32(5): p. 36575 .

25. Aguilar, H.N. and B.F. Mitchell, Physiological pathways and molecular mechanisms regulating uterine contractility. Hum Reprod Update, 2010. 16(6): p. 725-44.

26. Hashimoto, A., et al., Impact of endometriosis and adenomyosis on pregnancy outcomes. Hypertension Research in Pregnancy, 2019. 7(2): p. 50-55.

27. Graziano, A., et al., Diagnostic findings in adenomyosis: a pictorial review on the major concerns. Eur Rev Med Pharmacol Sci, 2015. 19(7): p. 1146-54.

28. Tellum, T., S. Nygaard, and M. Lieng, Noninvasive Diagnosis of Adenomyosis: A Structured Review and Meta-analysis of Diagnostic Accuracy in Imaging. J Minim Invasive Gynecol, 2019.

29. Champaneria, R., et al., Ultrasound scan and magnetic resonance imaging for the diagnosis of adenomyosis: systematic review comparing test accuracy. Acta Obstet Gynecol Scand, 2010. 89(11): p. 1374-84.

30. Van den Bosch, T. and D. Van Schoubroeck, Ultrasound diagnosis of endometriosis and adenomyosis: State of the art. Best Pract Res Clin Obstet Gynaecol, 2018. 51: p. 16-24.

31. Vercellini, P., et al., Adenomyosis and reproductive performance after surgery for rectovaginal and colorectal endometriosis: a systematic review and meta-analysis. Reprod Biomed Online, 2014. 28(6): p. 704-13.

32. Younes, G. and T. Tulandi, Effects of adenomyosis on in vitro fertilization treatment outcomes: a meta-analysis. Fertil Steril, 2017. 108(3): p. 483-490.e3.

33. Bianchi, P.H.M., et al., Extensive Excision of Deep Infiltrative Endometriosis before In Vitro Fertilization Significantly Improves Pregnancy Rates. Journal of Minimally Invasive Gynecology, 2009. 16(2): p. 174-180.

\section{Figures}




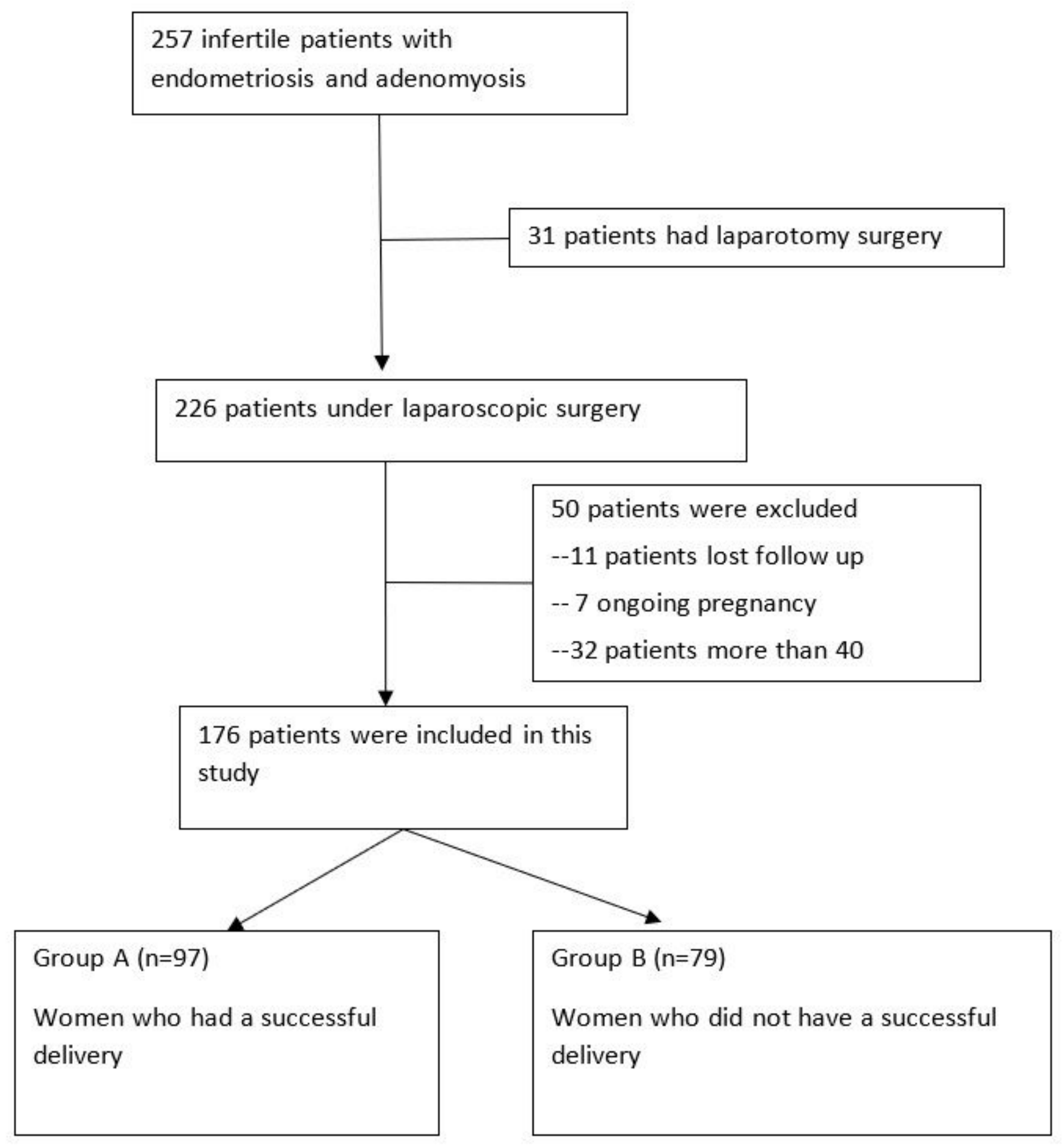

Figure 1

Flowchart of patients' selection 